\title{
Use of Clinical Decision Support to Improve Primary Care Identification and Management of Chronic Kidney Disease (CKD)
}

\author{
Cara B. Litvin, MD, MS, J. Madison Hyer, MS, and Steven M. Ornstein, MD
}

Background: Early detection of chronic kidney disease (CKD) can lead to interventions to prevent renal failure and reduce risk for cardiovascular disease, yet adherence to treatment goals is suboptimal in the primary care setting. The purpose of this study was to assess whether clinical decision support (CDS) can be used to improve the identification and management of CKD.

Methods: This 2 year demonstration study was conducted in 11 primary care PPRNet practices. CDS included a risk assessment tool, health maintenance protocols, flow chart and a patient registry. Practices received performance reports and hosted annual half day on-site visits.

Results: There were statistically significant increases in screening for albuminuria (median 24 month change $30 \%, p<0.0005$ ) and monitoring albuminuria (median 24 month change $25 \%, p<0.0005$ ). An absolute 23.5\% improvement in appropriate use of ACE-inhibitor or angiotensin receptor blocker and an absolute $7.0 \%$ improvement in hemoglobin measurement were not statistically significant. There were no clinical or statistically significant differences in other CKD CQMs. Facilitators to CDS use included practices' prioritization of improving CKD and staff use of standing orders. Barriers included incorporating use into existing workflow and variable use among providers.

Conclusions: Use of CDS to improve CKD identification and management in primary care practices shows promise. However, other barriers must be addressed to effectively achieve improvements in CKD outcomes. (J Am Board Fam Med 2016;29:604-612.)

Keywords: Albuminuria, Angiotensin Receptor Antagonists, Angiotensin-Converting Enzyme Inhibitors, Chronic Kidney Disease, Clinical Decision Support Systems, Hemoglobins, Hypertension, Practice-based Research, Primary Health Care, Risk Assessment, Workflow

The prevalence of chronic kidney disease (CKD) is increasing in the United States. ${ }^{1,2}$ In addition to be-

This article was externally peer reviewed.

Submitted 15 January 2016; revised 8 April 2016; accepted 13 April 2016.

From the Division of General Internal Medicine and Geriatrics, Department of Medicine, Medical University of South Carolina, South Carolina (CBL); the Department of Public Health Sciences, Medical University of South Carolina (JMH); and the Department of Family Medicine, Medical University of South Carolina (SMO).

Funding: This study was supported by the Agency for Health care Research and Quality (grant K08HS018984).

Prior presentation: Preliminary results from this study were presented at the Society of General Internal Medicine Annual Meeting on April 23, 2015 in Toronto, ON, Canada and have been published in abstract form.

Conflict of interest: none declared.

Disclaimer: The funding agency had no role in the study design; in data collection, analysis, or interpretation; in writing the report; nor in the decision to submit the article for publication. ing a risk factor for progression to end-stage renal disease, $\mathrm{CKD}$ is a risk factor for cardiovascular disease and all-cause mortality. ${ }^{3,4}$ The majority of patients with CKD are managed solely by primary care physicians, ${ }^{5,6}$ yet primary care adherence to clinical practice guidelines seems to be suboptimal. ${ }^{7-9}$ Interventions to improve the early identification and management of patients with CKD could reduce risks for the progression of renal disease and cardiovascular disease and have a major impact on public health. ${ }^{10}$

Inadequate recognition of patients with $\mathrm{CKD}$ and lack of awareness of treatment guidelines are 2 major barriers to providing quality care to patients

Corresponding author: Cara B. Litvin, MD, MS, Division of General Internal Medicine and Geriatrics, Medical University of South Carolina, 295 Calhoun St, Charleston, SC 29425 (E-mail: litvincb@musc.edu). 
Table 1. Characteristics of Participating Practices

\begin{tabular}{|c|c|c|c|c|c|}
\hline & State & Specialty & Providers & Clinical Staff Members & Patients per Clinician (Mean) \\
\hline 1 & WI & Family medicine & 2 MDs & $1 \mathrm{RN}, 3 \mathrm{MAs}$ & 933 \\
\hline 2 & $\mathrm{TN}$ & Family medicine & 2 MDs & 2 LPNs, 2 MAs & 1299 \\
\hline 3 & $\mathrm{CO}$ & Internal medicine & $1 \mathrm{MD}, 1 \mathrm{NP}, 1 \mathrm{PA}$ & $1 \mathrm{RN}, 2$ MAs & 2271 (assigned to MD only) \\
\hline 4 & WA & Family medicine & $1 \mathrm{MD}$ & $1 \mathrm{RN}$ & 521 \\
\hline 5 & NJ & Family medicine & $1 \mathrm{MD}$ & 0 & 636 \\
\hline 6 & $\mathrm{OH}$ & Internal medicine & 3 MDs & $1 \mathrm{RN}, 3 \mathrm{LPNs}, 2$ MAs & 886 \\
\hline 7 & PA & Family medicine & 4 MDs, 1 NP, 3 PAs & $5 \mathrm{RN}, 11 \mathrm{LPN}, 4$ MAs & 723 per MD; 884 per midlevel provider \\
\hline 8 & CT & Family medicine & 4 MDs & 4 MAs & 1041 \\
\hline 9 & $\mathrm{AZ}$ & Family medicine & $2 \mathrm{MDs}, 1 \mathrm{PA}$ & 3 MAs & 2032 per $\mathrm{MD} ; 959$ per $\mathrm{PA}$ \\
\hline 10 & $\mathrm{CA}$ & Family medicine & 3 MDs 3 PAs & 7 MAs & 1831 per $M D ; 147$ per $P A$ \\
\hline 11 & MI & Family medicine & $1 \mathrm{MD}$ & 4 MAs & 1629 \\
\hline 12 & MD & Family medicine & $1 \mathrm{MD}, 4 \mathrm{NPs}, 1 \mathrm{PA}$ & 4 MAs & 3061 per MD; 254 per midlevel provider \\
\hline
\end{tabular}

LPN, licensed practical nurse; MA, medical assistant; MD, medical or osteopathic doctor; NP, nurse practitioner; PA, physician assistant; RN, registered nurse.

with CKD. ${ }^{11}$ Electronic-health record (EHR)based reminders and clinical decision support (CDS) have been identified as potential tools to improve the identification of $\mathrm{CKD}$, facilitate monitoring, and improve adherence to treatment targets. To date, however, only a few small, single-site studies have evaluated the use of EHR-based tools to improve CKD care, ${ }^{12,13}$ and none have assessed provider and staff perceptions of their use. ${ }^{14,15}$

This 2-year demonstration study was designed to assess the impact of EHR-based CDS tools on a set of primary care CKD clinical quality measures (CQMs). We previously published the consensus process used to develop these CQMs. ${ }^{16}$ The purpose of this article is to present the impact of the intervention and describe facilitators and barriers to the use of the CDS tools.

\section{Methods}

\section{Study Practices}

The study was conducted within the Primary Care Practices Research Network (PPRNet), a national primary care practice-based research network, from September 2012 to September 2014. At the time of the study, all network members used a common EHR (Practice Partner; McKesson, San Francisco, CA). Using an automated extraction program, members regularly pool data for quality improvement and research. Twelve practices in 12 states, representing 25 physicians and 15 midlevel providers, volunteered to participate in the study in response to recruitment via the PPRNet listserv and announcements at an annual network meeting (Ta- ble 1). Practices with fewer than 50 patients with CKD were excluded. The study was approved by the institutional review board at the Medical University of South Carolina.

\section{CKD CDS Tools}

Several EHR-based CDS tools were developed by the research team to improve the identification and management of $\mathrm{CKD}$, including a CKD risk assessment tool, electronic health maintenance protocols, a CKD patient registry, and a CKD flowchart. The risk assessment tool (Figure 1) was designed to be embedded into general or disease-specific progress note templates for use by providers at the point of care. The tool prompted for at least annual estimated glomerular filtrate rate (eGFR) and urine albumin testing in patients with hypertension (HTN), diabetes mellitus (DM), or prior eGFR $<60 \mathrm{~mL} / \mathrm{min} / 1.73 \mathrm{~m}^{2}$. It also prompted the provider to diagnose CKD if a patient had an eGFR $<60 \mathrm{~mL} / \mathrm{min} / 1.73 \mathrm{~m}^{2}$. Additional prompts included a reminder of the patient's blood pressure goal and need for an angiotensin-converting enzyme inhibitor (ACEI) or angiotensin receptor blocker (ARB) based on the presence or absence of albuminuria, and a reminder for annual lipid and hemoglobin assessments for patients with a reduced eGFR.

Electronic health maintenance protocols were designed to be added to the EHR health maintenance tables. These protocols included prompts for eGFR, urine albumin, lipid, and hemoglobin testing when due based on customized rules (eg, eGFR 
Figure 1. Chronic kidney disease (CKD) risk assessment tool. ACEI, angiotensin-converting enzyme inhibitor; ARB, angiotensin receptor blocker; $\mathrm{BP}$, blood pressure; eGFR, estimated glomerular filtration rate; Hgb, hemoglobin; LDL, low-density lipoprotein.

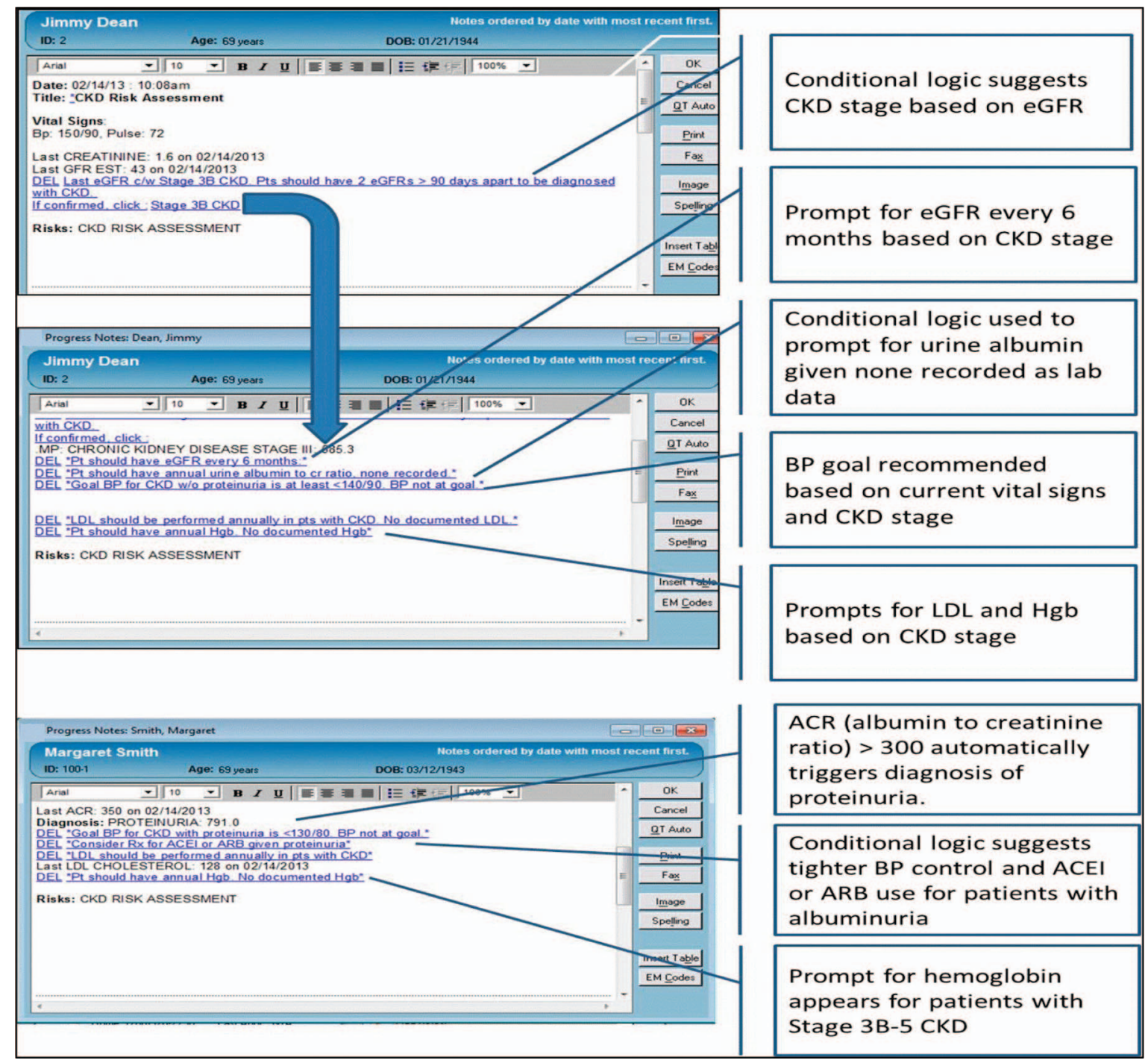

monitoring every 6 months for patients with a last eGFR between 30 and $60 \mathrm{~mL} / \mathrm{min} / 1.73 \mathrm{~m}^{2}$, hemoglobin monitoring every year for patients with a last eGFR $<45 \mathrm{~mL} / \mathrm{min} / 1.73 \mathrm{~m}^{2}$ ). These rules could be applied to both patients at-risk for CKD (patients with DM and/or HTN) and patients with CKD.

An EHR-based CKD flowchart (Figure 2) was designed to enable providers to concisely view CKD parameters over time. This was intended to help providers diagnose $\mathrm{CKD}$ in a patient with a persistently reduced eGFR, track the progression of CKD, and facilitate monitoring of associated risk factors and treatment targets.
A CKD patient registry (Figure 3) included all patients with DM, HTN, or CKD in the practice, along with relevant clinical, laboratory, and medication parameters (eg, most recent blood pressure, eGFR, date of last ACEI/ARB prescription). The registry also included lists of patients not meeting criteria for CKD CQMs.

\section{Intervention}

The study intervention, designed to facilitate the adoption of the CDS tools, included 2 half-day practice site visits annually and quarterly performance reports on the CKD CQMs. The first site visits were conducted during the first 2 months of 
Figure 2. Chronic kidney disease flowchart.

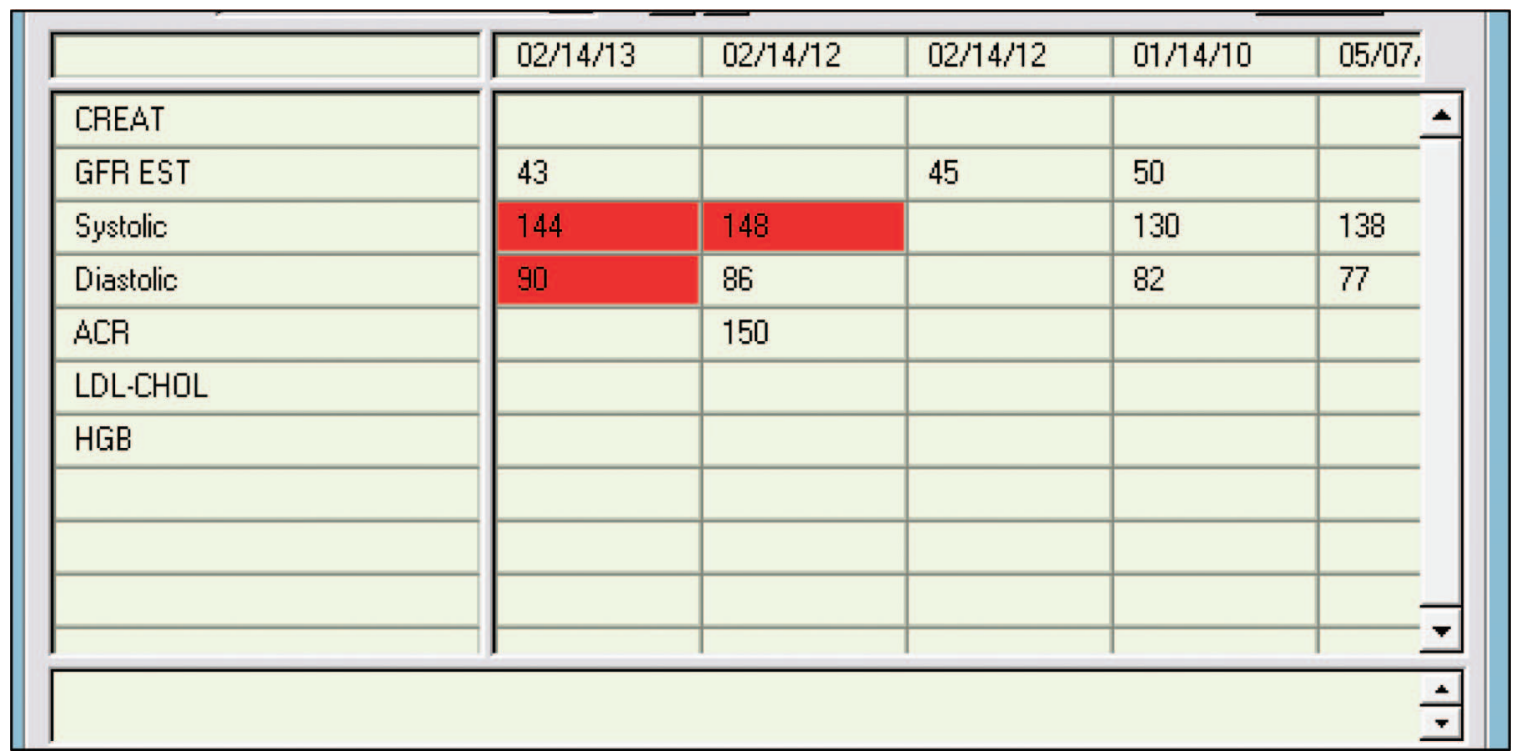

the study and the second during months 13 to 14 . Site visits were conducted by 1 or 2 members of the research team (CBL, SMO) and attended by the clinical staff at each practice. At the initial site visit, the CDS tools were introduced and installed at each practice and CDS training was provided. Guidelines for CKD identification and management were presented, ${ }^{3}$ and baseline performance on CKD CQMs was reviewed. Practices were then encouraged to make plans to facilitate use of the CDS tools to improve CKD care, such as implementing standing orders for clinical staff based on the health maintenance protocols or regularly reviewing the patient registry. At the second site visit, CKD performance was again reviewed and any reported problems with the CDS tools were addressed. Previous plans for improvement were reviewed and progress reported; additional plans were made based on each practice's individual needs. Between site visits, the research team contacted a representative from each practice via email quarterly to follow up on the practice's improvement plans. All participating practices continued the usual PPRNet procedure for submitting data extracts and received quarterly practice-level performance reports for each CKD CQM, along with an updated CKD patient registry.

\section{Study Measures and Outcomes}

Table 2 lists the CKD CQMs. All data were obtained from EHR extracts from participating prac- tices. Each CQM was calculated for active patients in each practice, defined as patients 18 years of age and older with a visit within the past year. For CQMs using eGFR, if not automatically calculated by a laboratory, eGFR was calculated using the Modification of Diet in Renal Disease equation, ${ }^{17}$ using each patient's age, sex, and race, if available. If a patient's race was not available, he or she was assumed to be nonblack for eGFR calculation. For CQMs applicable to patients with CKD, patients were considered to meet criteria for CKD if the most recent eGFR was $<60 \mathrm{~mL} / \mathrm{min} / 1.73 \mathrm{~m}^{2}$ and an eGFR $>90$ days before the most recent eGFR was also $<60 \mathrm{~mL} / \mathrm{min} / 1.73 \mathrm{~m}^{2}$, or if the patient had an albumin-to-creatinine ratio $>30 \mathrm{mg} / \mathrm{g}$. For CQMs assessing testing for albuminuria/proteinuria, spot urine albumin-to-creatinine ratio, 24hour urine albumin excretion, spot urine proteinto-creatinine ratio, and 24-hour urine protein excretion were considered appropriate tests. Macroalbuminuria/proteinuria was defined as a urine albumin-to-creatinine ratio $>300 \mathrm{mg} / \mathrm{g}, 24$-hour urine albumin $>300 \mathrm{mg}$, urine protein-to-creatinine ratio $>500 \mathrm{mg} / \mathrm{g}$, or 24 -hour urine protein $>500 \mathrm{mg}$.

\section{Statistical Analyses}

Mixed-effects regression models, controlling for baseline values and the number of eligible subjects, were used to assess change over time for each measure. For each measure, a quadratic model was 
Figure 3. Chronic kidney disease patient registry.

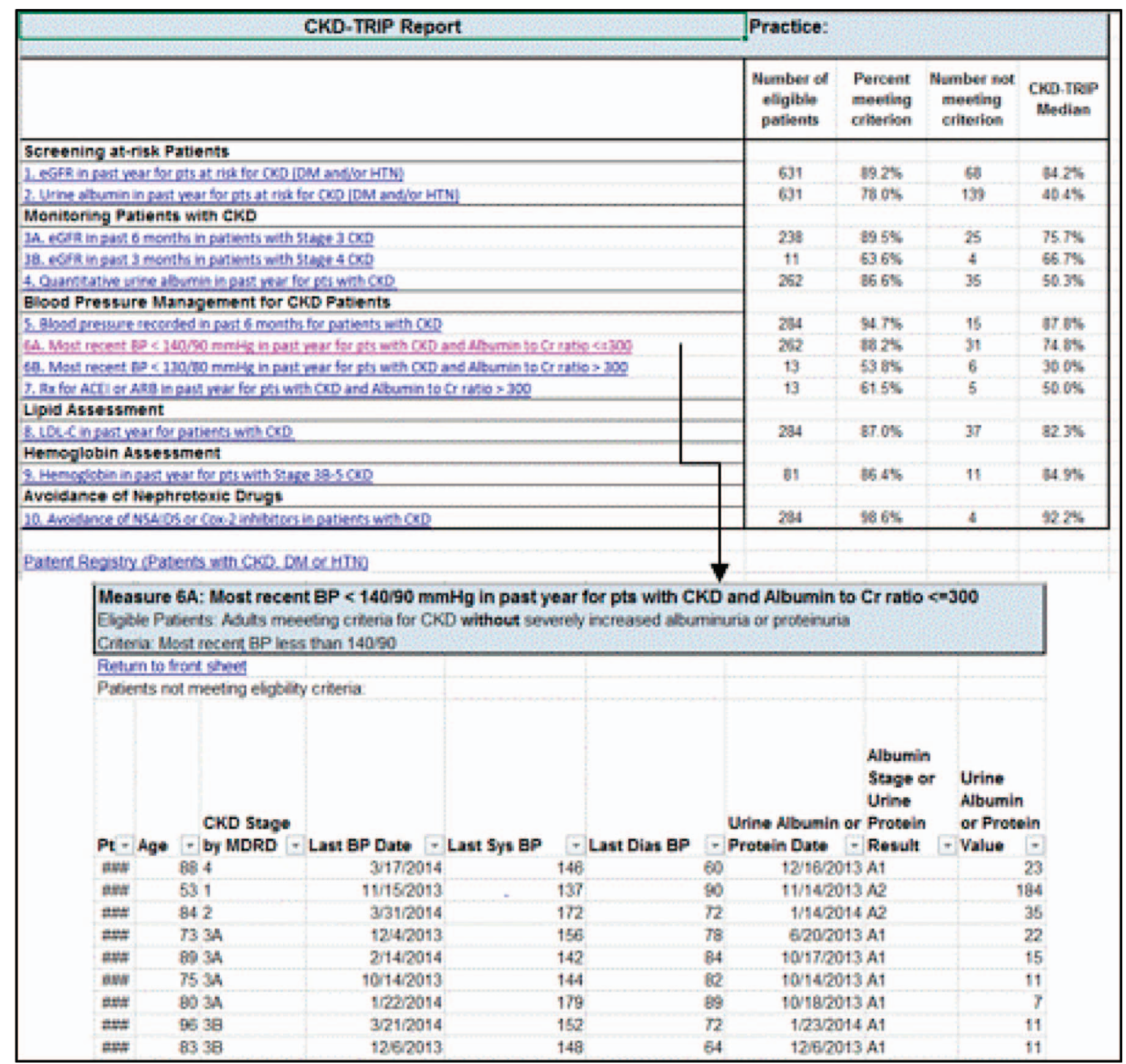

initially assessed for fit; for those without a significant quadratic term, a linear model was used. All statistical analyses were performed using SAS version 9.4 (SAS Institute, Inc., Cary, NC). $P$ values $<.05$ were considered statistically significant.

\section{Qualitative Data Collection and Analyses}

Detailed field notes were taken at each site visit to describe practice characteristics and document the practice's use of the CDS tools. To identify facilitators and barriers of CDS use for improving the identification and management of CKD, semistructured group interviews were conducted with all providers and staff at each practice during the second site visit. All qualitative data were re- viewed by the research team. CDS use as reported by providers was summarized across practices. To characterize facilitators and barriers to the use of CDS to improve CKD care, qualitative data were organized into 4 domains (provider, patient, organizational, and technical factors) based on a previously published framework used to assess CDS uptake. ${ }^{18,19}$

\section{Results}

Practice characteristics are described in Table 1, including the number of providers, the number of clinical staff members, and the average number of patients assigned to each clinician. Practices ranged 
Table 2. Performance on Chronic Kidney Disease Clinical Quality Measures over the 24-Month Intervention

\begin{tabular}{|c|c|c|c|c|c|}
\hline \multirow[b]{2}{*}{ CKD Clinical Quality Measure } & \multicolumn{2}{|c|}{ Baseline } & \multicolumn{2}{|c|}{24 Months } & \multirow{2}{*}{$\begin{array}{l}\text { Change from Baseline } \\
\text { to Month } 24 \\
\left(\%_{M 24-\mathrm{BL}}\right) \\
\end{array}$} \\
\hline & $\%_{\mathrm{BL}}$ & $\mathrm{N}_{\mathrm{BL}}$ & $\%_{\mathrm{M} 24}$ & $\mathrm{~N}_{\mathrm{M} 24}$ & \\
\hline \multicolumn{6}{|l|}{ Identification of patients with CKD } \\
\hline $\begin{array}{l}\text { eGFR in the past year for patients with } \\
\text { DM and/or hypertension }\end{array}$ & $87(80,93)$ & $929(590,1303)$ & $87.5(82,92)$ & $795(608,1256)$ & $0.5(-2.0,5.0)$ \\
\hline $\begin{array}{l}\text { Screening for albuminuria in the past year } \\
\text { for patients with DM and/or } \\
\text { hypertension }\end{array}$ & $21.5(16,26)$ & $929.5(590,1303)$ & $59(37,73)$ & $795(608,1256)$ & $30.0(23.0,46.0)^{*}$ \\
\hline \multicolumn{6}{|l|}{ Monitoring patients with CKD } \\
\hline $\begin{array}{l}\text { eGFR in the past } 6 \text { months for patients } \\
\text { with stage } 3 \text { CKD }\end{array}$ & $76(72,86)$ & $169.5(122,279)$ & $80.5(76,83)$ & $205.5(149,263)$ & $0.5(-3.0,3.0)$ \\
\hline $\begin{array}{l}\text { eGFR in the past } 3 \text { months for patients } \\
\text { with stage } 4 \text { CKD }\end{array}$ & $75(63,80)$ & $10.5(5,16)$ & $74(63,80)$ & $11.5(7,15)$ & $-3.0(-7.0,0.0)$ \\
\hline $\begin{array}{l}\text { Monitoring albuminuria in past year for } \\
\text { patients with CKD (without prior } \\
\text { macroalbuminuria or proteinuria) }\end{array}$ & $34.5(27,55)$ & $190.5(131,356)$ & $63(53,83)$ & $325(201,420)$ & $25.0(22.0,31.0)^{*}$ \\
\hline \multicolumn{6}{|l|}{ BP management in patients with CKD } \\
\hline $\begin{array}{l}\text { Most recent } \mathrm{BP}<140 / 90 \mathrm{mmHg} \text { for } \\
\text { patients with CKD without } \\
\text { macroalbuminuria or proteinuria }\end{array}$ & $76(68,83)$ & $191.5(131,357)$ & $76.5(73,82)$ & $325.5(201,422)$ & $2.5(0.0,7.0)$ \\
\hline $\begin{array}{l}\text { Most recent } \mathrm{BP}<130 / 80 \mathrm{mmHg} \text { for } \\
\text { patients with } \mathrm{CKD} \text { with } \\
\text { macroalbuminuria or proteinuria }\end{array}$ & $33(30,57)$ & $2.5(1,7)$ & $29(13,33)$ & $8.5(3,15)$ & $-1.5(-50.5,9.0)$ \\
\hline $\begin{array}{l}\text { ACEI or ARB in the past year for patients } \\
\text { with CKD and hypertension with } \\
\text { macroalbuminuria or proteinuria }\end{array}$ & $53.5(43,67)$ & $2.5(1,7)$ & $65.5(52,100)$ & $8.5(3,15)$ & $23.5(-5.5,43.5)$ \\
\hline \multicolumn{6}{|l|}{ Dyslipidemia in patients with CKD } \\
\hline $\begin{array}{l}\text { Lipid panel in the past year for patients } \\
\text { with CKD }\end{array}$ & $84.5(82,87)$ & $202.5(131,367)$ & $84(82,88)$ & $340.5(209,441)$ & $-0.5(-4.0,1.0)$ \\
\hline \multicolumn{6}{|l|}{ Anemia in patients with CKD } \\
\hline $\begin{array}{l}\text { Hemoglobin in the past year for patients } \\
\text { with eGFR }<45\end{array}$ & $77(74,82)$ & $60(34,110)$ & $86(70,91)$ & $69.5(39,91)$ & $7.0(0.0,8.0)$ \\
\hline \multicolumn{6}{|l|}{ Avoidance of potential nephrotoxic drugs } \\
\hline $\begin{array}{l}\text { Avoidance of NSAIDs or COX-2 } \\
\text { inhibitors in patients with CKD }\end{array}$ & $94(91,96)$ & $202.5(131,367)$ & $92(92,94)$ & $340.5(209,441)$ & $-1.0(-2.0,1.0)$ \\
\hline
\end{tabular}

Data are median (25th percentile, 75 th percentile).

${ }^{*} P<.0005$.

$\%_{\mathrm{BL}}$, Proportion of testing at baseline; $\%_{\mathrm{M} 24}$, proportion of testing at month $24 ; \%_{\mathrm{M} 24-\mathrm{BL}}$, absolute change in proportion; ACEI, angiotensin-converting enzyme inhibitor; $\mathrm{ARB}$, angiotensin receptor blocker; $\mathrm{CKD}$, chronic kidney disease; COX-2, cyclooxygenase 2; eGFR, estimated glomerular filtration rate; $\mathrm{DM}$, diabetes mellitus; $\mathrm{BP}$, blood pressure; $\mathrm{N}_{\mathrm{BL}}$, number of patients eligible for testing at baseline; $\mathrm{N}_{\mathrm{M} 24}$, number of patients eligible for testing at month 24; NSAID, nonsteroidal anti-inflammatory drug.

in size from 1 to 8 providers. One practice withdrew from the study after the initial site visit because of competing obligations. This practice's data were not included in the analysis. Another practice was unable to provide the final quarter of data because of a change in the extraction process. This practice's data were included in the analysis though July 1, 2014.

\section{Quantitative Results}

Table 2 presents both baseline and final (24 months) practice performance and change in performance for each CKD CQM. At baseline, median practice performance was relatively low $(<$ $35 \%$ ) for 3 of the CQMs (screening for albumin- uria for patients with DM and/or HTN, monitoring albuminuria in patients with CKD, and blood pressure $<130 / 80 \mathrm{mmHg}$ in patients with CKD and macroalbuminuria or proteinuria.) Median practice performance on ACEI or ARB use for patients with CKD and HTN with macroalbuminuria or proteinuria was modest $(53.5 \%)$, whereas median performance on all 7 other CQMs was $>75 \%$.

After 24 months, there were large statistically significant increases in 2 of the CQMs that were relatively low at baseline (screening for albuminuria for patients with DM and/or HTN and monitoring albuminuria in patients with $\mathrm{CKD}$ ). An absolute 23.5\% improvement in ACEI or ARB use for pa- 
Table 3. Reported Facilitators and Barriers to Use of Clinical Decision Support Tools to Improve Chronic Kidney Disease Management

\begin{tabular}{|c|c|c|}
\hline & Facilitators & Barriers \\
\hline Provider factors & $\begin{array}{l}\text { - CDS helps focus provider attention on } \\
\text { CKD } \\
\text { - Perception by users that CDS improves } \\
\text { care }\end{array}$ & $\begin{array}{l}\text { - Disagreement about CKD guidelines } \\
\text { - Confusion about CKD guidelines } \\
\text { - Concerns about data validity } \\
\text { - Patients comanaged by nephrologists } \\
\text { - Lack of awareness of CDS tools }\end{array}$ \\
\hline Organizational factors & $\begin{array}{l}\text { - Practice-wide prioritization of identifying } \\
\text { patients with CKD } \\
\text { - In-office urine collection and/or albumin } \\
\text { testing } \\
\text { - Standing orders for laboratory tests } \\
\text { - Patient registry used by staff for patient } \\
\text { outreach }\end{array}$ & $\begin{array}{l}\text { - Staff turnover } \\
\text { - Competing obligations (other incentive } \\
\text { programs) } \\
\text { - Failure to fully implement standing orders }\end{array}$ \\
\hline Technical factors & $\begin{array}{l}\text { - CDS tools customized to workflow of } \\
\text { practices by research team during site } \\
\text { visits } \\
\text { - Research team able to troubleshoot CDS } \\
\text { at site visits } \\
\text { - Research team demonstrated use of } \\
\text { patient registry during site visits }\end{array}$ & $\begin{array}{l}\text { - CDS tools required "extra clicks" } \\
\text { - Risk assessment tool did not always work } \\
\text { - Reports and tools did not capture labs ordered } \\
\text { by specialists } \\
\text { - Use of registry required re-identifying patients }\end{array}$ \\
\hline Patient factors & $\begin{array}{l}\text { - Patient education about CKD (including } \\
\text { educational handouts) } \\
\text { - Changing patients' expectations to adhere } \\
\text { to urine testing }\end{array}$ & $\begin{array}{l}\text { - Concern about patients seeing diagnosis of } \\
\text { CKD } \\
\text { - Concern about overdiagnosis of CKD }\end{array}$ \\
\hline
\end{tabular}

CKD, chronic kidney disease; CDS, clinical decision support.

tients with $\mathrm{CKD}$ and $\mathrm{HTN}$ with macroalbuminuria or proteinuria was noted but was not statistically significant, nor was an absolute $7.0 \%$ improvement in hemoglobin measurement for patients with an eGFR $<45 \mathrm{~mL} / \mathrm{min} / 1.73 \mathrm{~m}^{2}$. There were no clinical or statistically significant differences in other CKD CQMs.

\section{Qualitative Results}

The majority of providers reported using the CKD risk assessment tool, although the frequency of use varied by provider in several practices. All but 1 practice reported using health maintenance protocols. Six practices reported regularly reviewing the patient registry. No practices reported regular use of the flowchart.

Provider-reported facilitators and barriers to using CDS to improve CKD management are summarized in Table 3. Providers generally felt that use of these tools helped improve CKD management by focusing their attention on CKD, although there was occasional provider disagreement and confusion about the recommendations embedded in these tools. For example, some providers did not agree with monitoring albuminuria in patients already taking an ACEI or ARB. As another example, while providers generally agreed with avoiding nonsteroidal anti-inflammatory drug in patients with CKD, they sometimes they believed that the benefits to select patients occasionally outweighed the risks. Several providers reported that they did not monitor patients who were comanaged by nephrologists, thereby affecting performance on their monitoring CQMs. They also stated that nephrologists made changes in management that did not adhere to the CQMs (ie, taking patients off an ACEI or ARB). Some providers also reported overlooking some of the CDS tools available to them; no providers routinely used the CKD flowchart, and many providers reported forgetting that it was available.

Many practices reported prioritizing CKD improvement and implemented organizational changes to support use of the CDS tools for ordering tests, including adopting in-office urine albumin testing and establishing protocols for urine collection before office visits. Half of the practices used standing orders based on health maintenance protocols to empower staff to order appropriate laboratory tests. One third of practices used staff to review the patient registries and provide outreach to patients. In 2 practices, staff turnover was believed to impede the use of CDS tools. 
The CDS tools occasionally required modifications to work correctly at each practice. Some providers also felt that use of the tools required additional steps outside the existing workflow. At both site visits, the research team was able to make minor tweaks to the CDS tools to resolve any issues and ensure that they best accommodated practice workflow. A few practices reported that laboratory tests performed by specialists were not captured by the CDS tools.

Because these tools prompted providers to diagnose patients with $\mathrm{CKD}$, some providers noted concern about overdiagnosing patients with CKD and also reported that patients were concerned when seeing this diagnosis in an after-visit summary. A few practices began embedding links to CKD patient education in these tools, which they reported was favorably received.

\section{Discussion}

In this study we were able to successfully develop and implement CDS tools for CKD that were used by the 11 participating practices. Our intervention resulted in a dramatic increase in the number of patients at risk for and with CKD who received testing for albuminuria, a major prognostic indicator for cardiovascular disease, progression of disease, and death. ${ }^{3}$ Through this additional testing, practices participating in this study identified hundreds more patients meeting criteria for CKD who may benefit from early interventions, such as blood pressure control, use of an ACEI or ARB, and assessment of cardiovascular risk factors.

The intervention did not result in significant changes in performance on the other $9 \mathrm{CKD}$ CQMs. Clinically important improvements for 2 of these CQMs did not reach statistical significance because of heterogeneity among the practices, and baseline performance on 6 other CQMs was relatively high. One CQM with low performance at baseline did not improve (blood pressure $<130 / 80$ $\mathrm{mmHg}$ in patients with CKD and macroalbuminuria or proteinuria). However, the number of patients applicable for this measure increased throughout the course of the intervention as more patients were assessed for albuminuria, and the intervention may not have been long enough to achieve tight blood pressure control in these newly identified patients.

The qualitative evaluation provides some insights about how CDS tools may be effectively used in primary care practice. While the CKD risk assessment tool was designed to be used by providers to remind them of appropriate treatment targets such as blood pressure control, electronic health maintenance prompts were intended for use by clinical staff members in addition to providers. Many practices relied on clinical staff members to successfully operationalize the use of these prompts, particularly for urine albumin testing, for which there was significant improvement. These results suggest that some CDS tools, such as electronic reminders, may be more effective when operationalized by clinical staff rather than physicians. Other organizational changes adopted by practices, such as establishing in-office urine collection, likely also improved adherence to urine albumin testing, highlighting the importance of integrating CDS use with other practice processes. In addition, as described in the qualitative evaluation, while some reported barriers were directly related to use of the CDS tools, many were related to sociotechnical factors, such as provider disagreement with the recommendations included in the CDS tools, staff turnover, and patient education that were not addressed by the CDS-focused intervention.

To date, the few other studies of the use of CDS to improve CKD management in primary care have yielded mixed results. A prospective, nonrandomized study found that a CKD checklist incorporated into the EHR in 1 primary care clinic improved process CQMs such as testing for albuminuria and use of an ACEI/ARB, but not blood pressure control, consistent with the results of this study. ${ }^{12}$ In another single-center, cluster-randomized trial, automated reminders for primary care providers did not improve adherence to any outcomes, including assessment of albuminuria or blood pressure control. ${ }^{13}$ Similarly, a larger cluster-randomized trial of an enhanced laboratory prompt outside the EHR did not effectively improve adherence to CKD clinical practice guidelines. ${ }^{20}$

This study has several limitations. First, the study was conducted within a small group of practices that had volunteered to participate, without a control group. Furthermore, 2 practices did not complete the study. Second, in addition to CDS tools, study practices received academic detailing and performance reports, which may have affected CKD management and limits our ability to isolate the independent effects of the CDS tools. Third, there was considerable variability in practice char- 
acteristics including size, numbers and types of providers, and specialty. Finally, our outcomes included adherence to CKD CQMs, but our study was not designed to assess changes in blood pressure control, eGFR, or albuminuria, which could have important clinical relevance.

\section{Conclusion}

Despite these limitations, CDS tools show promise for improving the identification of patients with CKD in a group of diverse, "real-world" primary care practices, particularly when operationalized by clinical staff. However, organizational, provider, patient, and technical factors beyond the CDS tools themselves may affect whether they can be effectively used to improve care. For example, achieving improvements in CKD outcomes such as blood pressure control likely requires an additional focus on improving care coordination between primary care physicians and nephrologists and encouraging patient activation. ${ }^{21}$ Further comparison studies are needed to evaluate multifaceted interventions designed to combine CDS tools with these additional components to effectively improve CKD management in primary care.

The authors thank Paul J. Nietert, Ph.D. for assistance with this project and review of the manuscript. His time was funded, in part, by the National Institute of Health (NIH) Clinical and Translational Science Award program (NCATS Grant UL1TR001450).

\section{References}

1. Hoerger TJ, Simpson SA, Yarnoff BO, et al. The future burden of CKD in the United States: a simulation model for the CDC CKD Initiative. Am J Kidney Dis 2015;65:403-11.

2. Levey AS, de Jong PE, Coresh J, et al. The definition, classification, and prognosis of chronic kidney disease: a KDIGO Controversies Conference report. Kidney Int 2011;80:17-28.

3. KDIGO 2012 clinical practice guideline for the evaluation and management of chronic kidney disease. Kidney Int Suppl 2013;3:19-62.

4. Go AS, Chertow GM, Fan D, McCulloch CE, Hsu CY. Chronic kidney disease and the risks of death, cardiovascular events, and hospitalization. New Engl J Med 2004;351:1296-305.

5. Fox CH, Brooks A, Zayas LE, McClellan W, Murray B. Primary care physicians' knowledge and practice patterns in the treatment of chronic kidney disease: an Upstate New York Practice-based Research Network (UNYNET) study. J Am Board Fam Med 2006;19:54-61.
6. Philipneri MD, Rocca Rey LA, Schnitzler MA, et al. Delivery patterns of recommended chronic kidney disease care in clinical practice: administrative claims-based analysis and systematic literature review. Clin Exp Nephrol 2008;12:41-52.

7. Litvin CB, Nietert PJ, Wessell AM, Jenkins RG, Ornstein SM. Recognition and management of CKD in primary care. Am J Kidney Dis 2011;57:646-7.

8. Allen AS, Forman JP, Orav EJ, Bates DW, Denker BM, Sequist TD. Primary care management of chronic kidney disease. J Gen Intern Med 2011;26:386-92.

9. Tuot DS, Powe NR. Chronic kidney disease in primary care: an opportunity for generalists. J Gen Intern Med 2011;26:356-8.

10. Levey AS, Becker C, Inker LA. Glomerular filtration rate and albuminuria for detection and staging of acute and chronic kidney disease in adults: a systematic review. JAMA 2015;313:837-46.

11. Plantinga LC, Tuot DS, Powe NR. Awareness of chronic kidney disease among patients and providers. Adv Chronic Kidney Dis 2010;17:225-36.

12. Mendu ML, Schneider LI, Aizer AA, et al. Implementation of a CKD checklist for primary care providers. Clin J Am Soc Nephrol 2014;9:1526-35.

13. Abdel-Kader K, Fischer GS, Li J, Moore CG, Hess R, Unruh ML. Automated clinical reminders for primary care providers in the care of CKD: a small cluster-randomized controlled trial. Am J Kidney Dis 2011;58:894-902.

14. Fox C, Vassalotti J. Checklists as computer decision support at the point of care: a step forward in the recognition and treatment of CKD by primary care physicians. Clin J Am Soc Nephrol 2014;9:1505-6.

15. Narva AS. Decision support and CKD: not there yet. Clin J Am Soc Nephrol 2012;7:525-6.

16. Litvin CB, Ornstein SM. Quality indicators for primary care: an example for chronic kidney disease. J Ambul Care Manage 2014;37:171-8.

17. Levey AS, Coresh J, Greene T, et al. Using standardized serum creatinine values in the modification of diet in renal disease study equation for estimating glomerular filtration rate. Ann Intern Med 2006;145:247-54.

18. Moxey A, Robertson J, Newby D, Hains I, Williamson M, Pearson SA. Computerized clinical decision support for prescribing: provision does not guarantee uptake. J Am Med Inform Assoc 2010;17:25-33.

19. Sittig DF, Krall MA, Dykstra RH, Russell A, Chin HL. A survey of factors affecting clinician acceptance of clinical decision support. BMC Med Inform Decis Mak 2006;6:6.

20. Manns B, Tonelli M, Culleton B, et al. A cluster randomized trial of an enhanced eGFR prompt in chronic kidney disease. Clin J Am Soc Nephrol 2012;7:565-72.

21. Haley WE, Beckrich AL, Sayre J, et al. Improving care coordination between nephrology and primary care: a quality improvement initiative using the renal physicians association toolkit. Am J Kidney Dis 2015;65:67-79. 